\title{
VOLUME XXXII
}

\section{INDEX OF SUBJECTS AND AUTHORS}

\author{
Only main topics are listed, and reference is given to the \\ first page of each paper in which they are discussed
}

abortive lysogeny, 273

$\alpha-\mathrm{D}-\mathrm{N}$-acetylglucosaminidase, in mouse, 183

$\beta$-D-N-acetylglucosaminidase, in mouse, 183

acriflavine, effects in DNA repair, 19

Al-Murrani, W. K., 299

albino alleles, in mouse, 1

Anderson, Janice R., 135

anencephaly, in mouse, 135

antagonistic phenomena, 55

Apis pura

polymorphisms, 47

Archer, J. R., 79

Archer, James R., 183

Ascobolus immersus gene conversion, 67 $w$ loci, 67

Aspergillus nidulans choline locus, 309

assortative mating, in Ichneumonidae, 47

bacteriophage

$\lambda, 273$

pf 16,251

PR4, 207

sex specific, 207

Bainbridge, Brian W., 309

Barlow, J. A., 37

Beatty, R. A., 9

bentazone resistance in Nicotiana, 85

Bjerkandera adusta antagonistic phenomena, 55

blastocyst, triploid, 9

body size selection, in mouse, 299

Bombus americanorum polymorphism, 47

Bregliano, J. C., 259

Bucheton, A., 259

caffeine, effects on DNA repair, 19

Campbell, J. H., 273

Campbell, R. B., 151

Campostoma anomalum karyotype evolution, 37

Carlson, Peter S., 85

catabolic plasmids, 207, 251

Chapman, Verne M., 91 choline metabolism, 309

chromosomal evolution, 37

chromosome contamination, 113

conjugative plasmids, 25

Coriolus versicolor

genetic structure, $\mathbf{5 5}$

Coulter, V. J., 9

courtship behaviour, in Drosophila, 231

$p$-cresol degradation, 251

cyprinid fishes, evolution, 37

Deal, F. H., 37

deletion mapping, in mouse, 1

Diadromus pulchellus

polymorphisms, 47

diapause, in Lymantria dispar, 129

digynic triploidy, in rabbits, 9

diploids, ratchet mechanisms, 293

DNA polymerase, 25

DNA repair, 19

Drosophila melanogaster

behaviour in mazes, 231

courtship, 231

female lethal mutations, 103

female sterility, 113, 259

fractional mutations, 19

gene dosage effects, 103

hybrid dysgenesis, 259

insertion elements, 113

laterality, 231

linkage disequilibrium, 215

male mating advantage, 123

male recombination, 241

mutation, 259

ovary atrophication, 241

transformer locus, 103

Drosophila pseudoobscura malo mating advantage, 123

Dunn, H. M., 251

Dunn, N. W., 207, 251

dwarfism, in mouse, 195

Dykhuizen, D., 273

dysgenesis, 259

Ehrman, Lee, 231

Eicher, Eva M., 1

erythromycin resistance, 171 
Escherichia coli

bacteriophage, 207, 273

DNA polymerase I, 25

lysogeny, 273

plasmids, $25,207,251,273$

Evans, I. H., 171

exencephaly, in mouse, 135

Falconer, D. S., 195

female lethal locus, in Drosophila, 103

Formes cojanderi antagonistic phenomena, 55

Formica sp., polymorphisms, 47

fractional mutations, 19

$\alpha$-L-fucosidase, in mouse, 183

$\alpha$-D-galactosidase, in mouse, 183

$\beta$-D-galactosidase, in mouse, 183

gene conversion, control, 67

gene dosage effects, 103

gene rearrangement, 37

generalized symmetric selection, 151

Gluecksohn-Waelsch, Salome, 1

glycosidase, in mouse, 183

Gold, J. R., 37

growth hormone, in mouse, 195

gypsy moth, see Lymantria, 129

Heller, R., 293

Helmi, S., 67

heritability, 281

Hewetson, L., 251

$\beta$-D-hexoaminidase, in mouse, 183

Hill, William S., 281

Hisey, Brent N., 231

Hoy, Marjorie A., 129

Hyboganthus layi

karyotype evolution, 37

Hybopsis aestivalis

karyotype evolution, 37

Hybrid DNA, asymmetrical, 67

hymenoptera, polymorphisms, 47

hypopituitary dwarfism, 195

Ichneumonidae, polymorphisms, 47

infertility, in mouse, 79

inhibition of repair, 19

insertion elements, in Drosophila, 113

Johnson, F. M., 215

Karlin, Samuel, 151

karyotype evolution, 37

Kidwell, M. S., 259

Knights, P. J., 135

Lamb, B. C., 67
Langley, Charles H., 215

Lasioglossum zephyrum polymorphisms, 47

laterality, in Drosophila sp., 231

linkage disequilibrium, 215

Lorkiewicz, Zbigniew, 319

Lymantria dispar diapause, genetic bases, 129

Lynch, Carol Becker, 129

lysogeny, 273

male mating advantage, in Drosophila, 123

male recombination, in Drosophila, 241

malic enzyme, mitochondrial, 1

Mandel, S. K., 19

$\alpha$-D-mannosidase, in mouse, 183

Markham, Paul, 309

Markow, Therese A., 123

Marshall, Terri, 103

maternal effects, in mouse, 299

maze behaviour, in Drosophila, 231

migration, effects on polymorphisms, 151

misrepair of DNA, 67

mitochondria, malic enzyme, 1

mitomycin C, 251

mosaics, X-chromosome, 91

Mukherjee, A. S., 19

Muller's ratchet, 293

multi-deme populations, 151

multi-locus selection, 151

Mus musculus

albino alleles, 1

body size, 299

curly tail, 135

exencephaly, 135

glucosidase, 183

growth hormone, 195

hypopituitary dwarfism, 195

infertility, 79

liver enzymes, 183

malic enzyme, 1

maternal effects, 299

mitochondria, 1

phosphoglycerate kinase, 91

pseudencephaly, 135

spermatozoal enzymes, 183

spina bifida, 135

$t$-complex, 79

mutator genes, 171

negative heterosis, 47

Neurospora crassa

choline metabolism, 309

gene conversion, 67

Nicotiana tabacum

herbicide tolerance, 85

nitrogen fixation, 319 
nodulation, 319

non-dysjunction, 259

non-epistatic selection, 151

non-mendelian inheritance, 113,259

Notropis umbratilis

karyotype evolution, 37

oligomycin resistance, 171

Oryctolagus cunniculus

digynic triploidy, 9

ovary atrophication, in Drosophila, 241

Pasteur, Nicole, 47

Pélisson, A., 113, 259

penetrance, 135

Perelle, Ira, 231

petite mutants, of yeast, 171

Phaeolus schweinitzii

antagonistic phenomena, 55

Phenacobius mirabilis

karyotype evolution, 37

phenmedipharm resistance, 85

phosphoglycerate kinase polymorphism, 91

Picard, G., 259

Pidduck, Heather G., 195

Pimephales vigilax

karyotype evolution, 37

plasmid

copy number, 25

NAH, 207

nodulation controlling, 319

pND2, 207

RZ, 207

R6K, 25

TOC, 207

Pogonomyrnex sp., polymorphisms, 47

population cages, size effects, 215

post-natal maternal effects, 299

procatechuate degradation, 251

pseudencephaly, in mouse, 135

Pseudomonas aeroginosa

plasmids, 25, 207, 251

Pseudomonas putida

plasmids, 207

rabbit, see Oryctolagus

Radin, David N., 85

ratchet mechanisms, 293

Rayner, A. D. M., 55

recombination, 293 repair synthesis, 19

replication of DNA, 25

rex mutations of phage $\lambda, 273$

Rhizobium trifolii

non-nodulating mutants, 319

plasmids, 319

Richardsonius egregius

karyotype evolution, 37

Roberts, R. C., 299

Rojas-Rousse, Danielle, 47

Rolfe, B. S., 273

Saccharomyces cerevisiae

mutator genes, 171

petite mutants, 171

Schizophyllum

gene conversion, 67

Self, Steven J., 79, 183

selfing, 293

Shaumar, Nagat, 47

Smith, Diana B., 215

Smith, J. Maynard, 293

Smith, J. T., 25

Solenopsis sp., male gene inactivation, 47

speciation, $\mathbf{3 7}$

spermatocytes, mutagenesis, 19

spina bifida, in mouse, 135

$t$-complex, of mouse, 79

Thompson, James N., 231

tobacco, see Nicotiana

Todd, N. K., 55

transformer locus, in Drosophila, 103

Turchin, Howard A., 1

Tweats, D. J., 25

Wallace, Margaret E., 135

West, John D., 91

White, G. P., 207

Whittle, J. R. S., 103

Wilkie, D., 171

Winchester, B. S., 79, 183

Womax, W. D., 37

$\mathrm{X}$-ray mutagenesis, 1,19

Yannopoulus, George, 241

yeast, see Saccharomyces, 171

Zurkowski, Witold, 319 